\title{
Hydrolytic enzyme production by Clostridium difficile and its relationship to toxin production and virulence in the hamster model
}

\author{
S. V. SEDDON, I. HEMINGWAY and S. P. BORRIELLO*
}

\section{Microbial Pathogenicity Research Group, Division of Communicable Diseases, MRC Clinical Research Centre, Watford Road, Harrow, Middlesex HA1 3UJ}

\begin{abstract}
Summary. Thirty isolates of Clostridium difficile expressing different degrees of toxigenicity and virulence in an animal model were assayed for the production of chondroitin-4-sulphatase, hyaluronidase, heparinase, collagenase and protease. All strains demonstrated some hydrolytic enzyme activity. There was no direct correlation between toxigenic status, or virulence, and hydrolytic enzyme production. However, all five strains known to be highly virulent in the hamster model had hyaluronidase, chondroitin-4-sulphatase and collagenase activity whereas only three of five toxigenic but poorly virulent strains had these activities, the collagenase activity being weak in all three cases. The only two proteolytic strains are also highly virulent. The potential tissue damaging properties of these hydrolytic enzymes may help to explain the differences in virulence of $C$. difficile strains seen in the Syrian hamster model of antibiotic-associated colitis, and may contribute to the spectrum of disease seen in man. It is also possible that chondroitin-4-sulphatase, hyaluronidase and collagenase activity may release essential nutrients, promoting establishment of $C$. difficile in the gut.
\end{abstract}

\section{Introduction}

Clostridium difficile is the major cause of pseudomembranous colitis and is implicated in many cases of antibiotic-associated colitis and diarrhoea (George et al., 1978; Larson et al., 1978; Borriello and Larson, 1981). The pathogenicity of the organism has been explained in part by the identification of several known and putative virulence factors - toxin production (Taylor et al., 1981; Libby and Wilkins, 1982), adhesion to gut mucosa (Borriello et al., 1988b), expression of fimbriae (Borriello et al., 1988a), capsule production (Borriello et al., in press) and hydrolytic enzyme production (Hafiz, 1974). Although considerable effort has been made in the study of $C$. difficile toxins and the adhesive properties of the organism, little work has been reported on the production by $C$. difficile of hydrolytic enzymes. A wide range of hydrolytic enzymes is produced by several species of anaerobic bacteria and there is evidence that these enzymes may play a role in disease processes (Steffen and Hentges, 1981). In the present study,

Received 12 June 1989; revised version accepted 23 Aug. 1989.

* Correspondence should be sent to Dr S. P. Borriello. we examined the extent of hydrolytic enzyme production by $C$. difficile isolates, in particular those of known toxigenicity and virulence.

\section{Materials and methods}

\section{C. difficile strains and growth conditions}

Thirty strains of $C$. difficile were examined: 19 were toxigenic strains (eight from adults, six from infants, three from animals, one from the environment and strain VPI 10463 originally isolated from an abscess) and 11 were non-toxigenic (eight from adults, two from infants and one from an animal). Ten of the toxigenic strains (table I) represented strains of differing virulence in the hamster animal model of disease and nine of these have been described in detail elsewhere (Borriello et al., 1987).

All strains were grown on Brain Heart Infusion (BHI) Agar (Difco) supplemented with horse blood $5 \%$, at $37^{\circ} \mathrm{C}$ in an atmosphere of $\mathrm{N}_{2} 80 \%, \mathrm{CO}_{2} 10 \%, \mathrm{H}_{2} 10 \%$ in an anaerobic cabinet (Forma Scientific, Marietta, $\mathrm{OH}$, USA). Innocula for enzyme assays were taken from 48-h cultures. In all cases but one, a single heavy inoculation with a loop was used. For one of the spectrophotometric assays (see below) the cells were resuspended in phosphate buffered saline-A (PBSA) to an optical density of approximately 0.5 at $580 \mathrm{~nm}$. 


\section{Hydrolytic enzyme assays}

Chondroitin-4-sulphatase and hyaluronidase activities. These were determined by an agar-plate method (Tipler and Embery, 1985) and by two spectrophotometric assays (Aminoff et al., 1952; Yamagata et al., 1968). Briefly, for the plate method, filter-sterilised substrate, either hyaluronic acid or chondroitin-4-sulphate (Sigma), was added to autoclaved BHI agar to a final concentration of $400 \mu \mathrm{g} /$ $\mathrm{ml}$, and filter sterilised bovine serum albumin (fraction $\mathrm{V}$, Sigma) was added to a final concentration of $1 \% \mathrm{w} / \mathrm{v}$. Inoculated plates were incubated for $72 \mathrm{~h}$ anaerobically and then flooded with $2 \mathrm{M}$ acetic acid. After $10 \mathrm{~min}$, the non-degraded substrate polymers precipitate as a complex with serum albumin, whereas a clear zone remains around those colonies that produce hyaluronidase and chondroitin-4-sulphatase.

For the first spectrophotometric assay, BHI broth containing test substrate $400 \mu \mathrm{g} / \mathrm{ml}$ was dispensed in 3$\mathrm{ml}$ volumes into $7-\mathrm{ml}$ screw-capped bottles, inoculated with a loopful of growth from a 48-h BHI-blood agar culture and incubated anaerobically for up to $72 \mathrm{~h} ; 1-\mathrm{ml}$ samples were removed every $24 \mathrm{~h}$. The samples were microcentrifuged at $13000 \mathrm{rpm}$ and $20 \mu$ of supernate was added to $4 \mathrm{ml}$ of distilled water. The UV absorbance at $232 \mathrm{~nm}$ was read for each substrate against a noninoculated (negative control) broth. Those tests that produced a UV absorbance equal to or greater than that produced by Porphyromonas (Bacteroides) gingivalis (positive control) were recorded as positive. This assay detects the first step in the breakdown of these substrates, i.e., the release by a lyase of unsaturated disaccharidecontaining intermediates.

The second spectrophotometric assay detected the presence of $\mathrm{N}$-acetylhexosamine sugars, final breakdown products of chondroitin and hyaluronic acid (Aminoff $e t$ $a l ., 1952)$. This assay was designed to detect the presence of preformed enzymes. A $0.5-\mathrm{ml}$ volume of an aqueous $0.1 \% \mathrm{v} / \mathrm{v}$ solution of substrate was added to $2.5 \mathrm{ml}$ of $0.1 \mathrm{M}$ sodium acetate buffer ( $\mathrm{pH} \mathrm{6.4)}$ ) and $0.5 \mathrm{ml}$ of a suspension in PBSA of a 48-h BHI agar culture of $C$. difficile was added. The reaction mixture was incubated aerobically at $37^{\circ} \mathrm{C}$, for $4 \mathrm{~h}$, and $0.5 \mathrm{ml}$ was then added to $0.1 \mathrm{ml}$ of $0.8 \mathrm{M}$ potassium tetraborate $(\mathrm{pH} \mathrm{9.2)}$. The resulting solution, in which there was a vigorous reaction, was transferred to a boiling water bath for $3 \mathrm{~min}$ and cooled under tap water, after which $3 \mathrm{ml}$ of dimethyl aminobenzaldehyde (DMAB) solution (10 g of DMAB dissolved in $100 \mathrm{ml}$ of glacial acetic acid containing $10 \mathrm{M}$ $\mathrm{HCl} 12.5 \% \mathrm{v} / \mathrm{v}$ ), was added to the solution and mixed. The mixture was then incubated for $20 \mathrm{~min}$ at $37^{\circ} \mathrm{C}$ before final cooling under running tap water. The absorbance was then determined at $585 \mathrm{~nm}$ and the result obtained by comparison with a standard curve of $\mathrm{N}$ acetylglucosamine versus absorbance.

The ability of mixed cultures of isolates to degrade hyaluronic acid and chondroitin-4-sulphate was also investigated. BHI broth containing test substrate $400 \mu \mathrm{g} /$ $\mathrm{ml}$ was inoculated with various combinations of isolates and incubated for up to $120 \mathrm{~h} ; 1-\mathrm{ml}$ samples were removed every $24 \mathrm{~h}$ and assayed by the first spectrophotometric assay.

Heparinase activity. This was assayed by the method of Riley (1987). Heparin obtained as a sodium salt (Sigma) was diluted in distilled water to a final concentration of $5 \mathrm{U} / \mathrm{ml}$. The solution was sterilised by membrane filtration $(0.45 \mu \mathrm{m})$ before dispensing $20-\mu \mathrm{l}$ volumes into wells of a microtitration plate; each well contained $30 \mu$ of the PBSA suspension of $C$. difficile. After incubation anaerobically overnight at $37^{\circ} \mathrm{C}, 20 \mu \mathrm{l}$ of aqueous toluidine blue (Sigma) $0.01 \%$ was added to each well. A positive reaction was indicated by a blue colour, whereas for a negative reaction the solution remained pink.

Collagenase activity. A simple agar-plate technique was used to screen for collagenase activity, and, in selected cases, a more sensitive spectrophotometric assay was also used. Collagen type 1 was extracted and purified from rat tails (Bornstein, 1958) and sterilised by UV irradiation for $30 \mathrm{~min}$ before incorporation into $\mathrm{BHI}$ agar at a final concentration of $1 \%$. The medium was inoculated and incubated for $72 \mathrm{~h}$, and then flooded with aqueous amido black (Sigma) $0.01 \%$. Weak blue staining indicated a positive result and dark staining a negative result.

The more sensitive spectrophotometric assay relies on the detection of hydroxyproline, one of the breakdown products of collagen. UV-sterilised collagen was dissolved in BHI broth to a final concentration of $1 \%$. Cultures were incubated anaerobically at $37^{\circ} \mathrm{C}$ and $1-\mathrm{ml}$ samples were removed before and at 24 and $48 \mathrm{~h}$ after inoculation. To each sample was added $1 \mathrm{ml}$ each of $0.01 \mathrm{M}$ aqueous copper sulphate solution, $2.5 \mathrm{M}$ sodium hydroxide and $\mathrm{H}_{2} \mathrm{O}_{2} 6 \%$. The reaction mixtures were shaken occasionally for $5 \mathrm{~min}$ before being placed in a water bath at $80^{\circ} \mathrm{C}$ for a further $5 \mathrm{~min}$, then cooled under running tap water and $4 \mathrm{ml}$ of $1.5 \mathrm{M}$ sulphuric acid was added with agitation followed by $2 \mathrm{ml}$ of DMAB ( $5 \% \mathrm{w} / \mathrm{v}$ in iso-propanol) solution, again with thorough mixing. The solution was placed in a water bath at $70^{\circ} \mathrm{C}$ for $16 \mathrm{~min}$ and finally cooled under running tap water. The absorbance was recorded at $490 \mathrm{~nm}$ and the result obtained by comparison with a standard curve of hydroxyproline concentration versus absorbance at $490 \mathrm{~nm}$. C. histolyticum NCTC 1234 was used as a positive control. For the purpose of interpretation, a value $40 \mu \mathrm{mol}$ of hydroxyproline released/mg of protein after $48 \mathrm{~h}$ (i.e., at least half of that produced by $C$. histolyticum) was considered to be a positive result. Anything less than this value represented minimal, though possibly still significant, activity.

\section{Protease activity}

A simple agar-plate technique was used to screen for non-specific proteolytic activity. Gelatin (Sigma) and skimmed milk powder (Oxoid) were made up as aqueous $10 \%$ solutions and boiled for $10 \mathrm{~min}$ before being added to autoclaved Columbia Agar Base (Oxoid) to a final concentration of $1 \%$. The plates were inoculated and incubated for $72 \mathrm{~h}$. The gelatin plates were then flooded with aqueous amido black (Sigma) $0.01 \%$. Clearing around colonies in the opaque skimmed milk plates, and 
weak blue staining in the gelatin plates, were recorded as positive results. $P$. gingivalis was used as a positive control.

\section{Results}

\section{Chondroitin-4-sulphatase and hyaluronidase activity}

The ability of the strains of $C$. difficile to degrade these two bio-polymers varied. Ten of the 19 toxigenic strains and five of the 11 non-toxigenic strains degraded both hyaluronic acid and chondroitin-4-sulphate (tables I and II). Interestingly, all five of the strains known to be highly virulent in the hamster model of disease could degrade these substrates whereas only three of the five poorly virulent toxigenic strains could (table I). In addition, four strains (three toxigenic and one nontoxigenic) could partially degrade both substrates (table II), as indicated by a negative plate assay but the build up of disaccharide intermediates in the spectrophometric assay. All of the strains that could only partially degrade these substrates were isolated from infants.

The results of assays for hyaluronic acid and chondroitin-4-sulphate hydrolysis were identical for each isolate; therefore, since hyaluronic acid is far more expensive and difficult to solubilise, chondroitin-4-sulphate was used routinely to test strains in the second spectrophotometric assay. Analysis of the breakdown of unsaturated disaccharide-containing intermediates, by the second spectrophotometric assay, to $\mathrm{N}$-acetylhexosamine sugars confirmed that these isolates from infants appeared to be incapable of degrading the disaccharide intermediate because little $\mathrm{N}$-acetylhexosamine sugars were detected (table III). The more virulent strains $2 B$ and $P T$ demonstrated relatively high specific activities in this assay. The resultant accumulation of the intermediate disaccharide by strain DY that could only partially degrade chondroitin-4-sulphate was reduced on co-cultivation with strain 2B which could fully degrade both compounds resulting in the release of the $\mathrm{N}$ acetylhexosamine (figure). Co-cultivation of a negative isolate (strain $\mathrm{KN}$ ) with a strain capable of partial hydrolysis releasing the disaccharide-containing intermediates (strain DY) yielded the same breakdown pattern of strain DY alone (figure). The other three infant strains (BR, PI and RY) capable of only partial degradation yielded results similar to those shown in the figure when co-cultivated with strains $2 B$ or KN. Similar results were obtained for a selection of strains (VPI, 2B DY, KN) assayed with hyaluronic acid, confirming the equal susceptibility of the two glycosaminoglycans to hydrolysis.

\section{Collagenase activity}

Only three isolates of $C$. difficile yielded a definite positive result by the plate assay (table I). All three were toxigenic strains which were highly virulent in the hamster model. Two of the strains, P-1 and $2 \mathrm{~B}$, and a selection of 10 of some of those that give negative or weak results for collagenase in the plate assay, including two toxigenic strains of low virulence (strains DD and Bat), were screened spectrophotometrically for collagenase activity. All

Table I. Hydrolytic enzyme production by toxigenic isolates of C. difficile of known virulence

\begin{tabular}{|c|c|c|c|c|c|c|c|}
\hline \multirow[b]{2}{*}{ Strain } & \multirow[b]{2}{*}{ Virulence* } & \multirow[b]{2}{*}{ Source } & \multicolumn{5}{|c|}{ Enzyme activity } \\
\hline & & & HA & $\mathrm{C} 4 \mathrm{~S}$ & Hep & Coll & Prot \\
\hline $2 \mathrm{~B}$ & High & Animal & + & + & W & + & + \\
\hline P-1 & High & Adult & + & + & + & + & - \\
\hline B-1 & High & Adult & + & + & + & W & - \\
\hline PT & High & Adult & + & + & W & $\mathbf{W}$ & + \\
\hline VPI 10463 & High & Abscess & + & + & + & + & - \\
\hline $\operatorname{Tra} 5 / 5$ & Low & Infant & + & + & + & W & - \\
\hline $\mathrm{SC}$ & Low & Animal & + & + & + & W & - \\
\hline DD & Low & Animal & - & - & + & - & - \\
\hline MA & Low & Infant & + & + & + & W & - \\
\hline Bat & Low & Environment & - & - & w & - & - \\
\hline
\end{tabular}

+ , Positive; W, weak; - , negative; HA, hyaluronidase; C4S, chrondroitin4-sulphatase; Hep, heparinase; Coll, collagenase; Prot, protease.

* In the Syrian hamster model (Borriello et al., 1987). 
Table II. Hydrolytic enzyme production by various $C$. difficile isolates of known toxigenicity

\begin{tabular}{lccccccc}
\hline & & & \multicolumn{5}{c}{ Enzyme activity } \\
\cline { 3 - 8 } Strain & $\begin{array}{c}\text { Toxigenic } \\
\text { status }\end{array}$ & Source & HA & C4S & Hep & Coll & Prot \\
\hline FN & + & Adult & - & - & + & - & - \\
HY & + & Adult & - & - & + & - & - \\
NS & + & Adult & + & + & W & W & - \\
OE & + & Adult & + & + & W & W & - \\
TT & + & Adult & - & - & W & - & - \\
DY & + & Infant & P & P & + & - & - \\
BR & + & Infant & P & P & W & W & - \\
PE & + & Infant & - & - & + & - & - \\
PI & + & Infant & P & P & + & + & - \\
M-I & - & Adult & + & + & + & - & - \\
PN & - & Adult & + & + & + & - & - \\
WS & - & Adult & + & + & + & W & - \\
PR & - & Adult & - & - & W & - & - \\
BE & - & Adult & - & - & W & - & - \\
MN & - & Adult & + & + & + & W & - \\
PT-1 & - & Adult & - & - & - & - & - \\
WR & - & Adult & - & - & - & - & - \\
S-1 & - & Dog & + & + & + & W & - \\
KN & - & Infant & - & - & + & - & - \\
RY & - & Infant & P & P & W & - & - \\
& & & & & & & -
\end{tabular}

+ , Positive; - , negative; W, weak; $\mathbf{P}$, partial hydrolysis (negative plate assay, disaccharide accumulation); HA, hyaluronidase; Hep, heparinase; C4S, chondroitin-4-sulphatase; Coll, collagenase; Prot, protease.

Table III. Breakdown of disaccharide-containing intermediates (released from chondroitin-4-sulphate) to $\mathrm{N}$-acetylgalactosamine

\begin{tabular}{llcc}
\hline Strain & Source & $\begin{array}{c}\text { Hydrolysis of } \\
\text { chondroitin-4- } \\
\text { sulphate }\end{array}$ & $\begin{array}{c}\text { N-acetylgalactosamine } \\
\text { released after 48h } \\
\text { (mmol/mg of protein) }\end{array}$ \\
\hline 2B & Animal & + & 2.00 \\
PT & Adult & + & 1.20 \\
NS & Adult & + & 1.04 \\
MN & Adult & + & 0.86 \\
M-1 & Adult & + & 0.78 \\
RY & Infant & P & 0.42 \\
DY & Infant & P & 0.40 \\
BR & Infant & P & 0.36 \\
DD & Animal & - & 0.28 \\
KN & Infant & - & 0.26 \\
PI & Infant & P & 0.20 \\
Bat & Infant & - & 0.15 \\
\hline
\end{tabular}

+ , Positive; - , negative; $P$, partial hydrolysis.

strains showed some activity, though in general the infant strains were less active (table IV). The two strains known to be highly virulent in the hamster model (strains 2B and P-1) showed the greatest collagenase activity.

\section{Heparinase and protease activity}

Heparinase activity was detected in all but two strains. For detection of protease activity, the gelatin plates proved difficult to read even when 


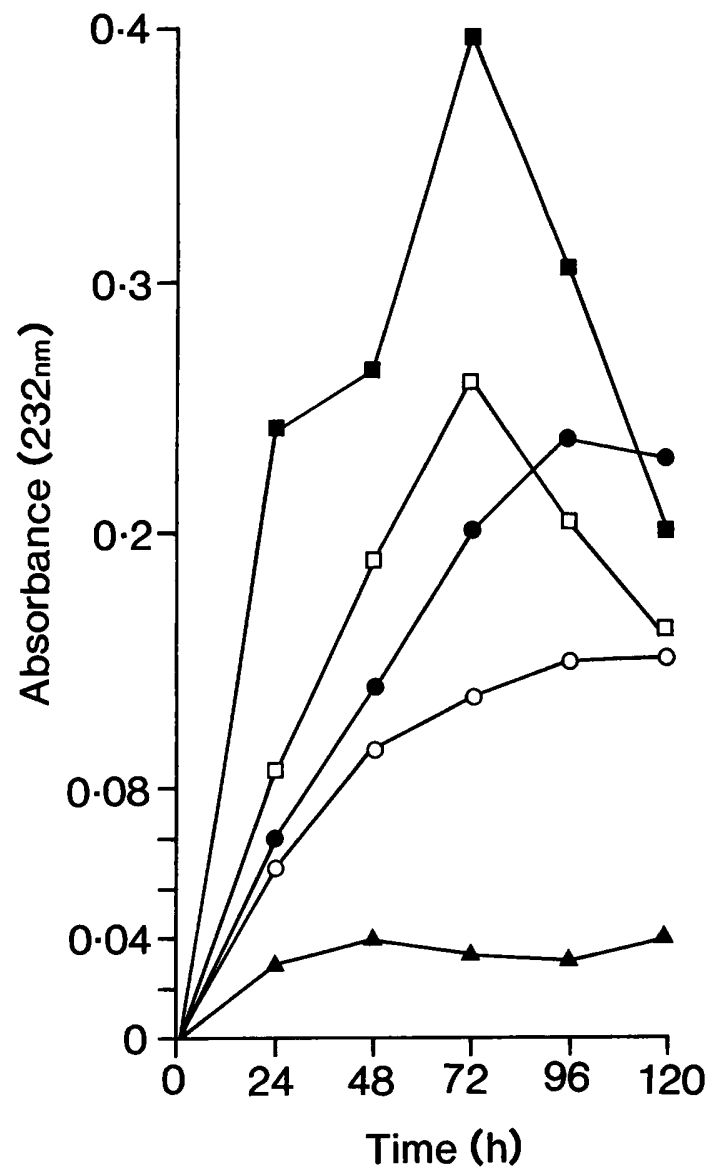

Figure. The release by $C$. difficile of disaccharide intermediates from glucosaminoglycans as detected by UV absorbance at 232nm: $\square, 2 \mathrm{~B}$ (full hydrolysis); $\square, 2 \mathrm{~B}+\mathrm{DY} ; \boldsymbol{\Delta}, \mathrm{KN}$ (no hydrolysis);, $\mathrm{DY}$ (partial hydrolysis); O, KN + DY.

stained with toluidine blue. The more opaque skimmed-milk plates showed distinct zones of clearing. There was, however, detectable protease activity for only two isolates (strains $2 \mathrm{~B}$ and PT); both of these were toxigenic strains that were highly virulent in the hamster model (table I).

\section{Discussion}

The results show clearly that $C$. difficile is capable of producing a variety of hydrolytic enzymes. The distribution of hydrolytic enzyme production does not directly correlate with toxin production. The production of chondroitin-4-sulphatase, hyaluronidase, collagenase and protease, however, does appear to have some association with virulence. The most highly virulent isolates, as determined in the Syrian hamster model (Borriello et al., 1987),
Table IV. Release of hydroxyproline as a measure of collagenase activity

\begin{tabular}{llcc}
\hline Strain & Source & $\begin{array}{c}\text { Collagenase } \\
\text { plate assay }\end{array}$ & $\begin{array}{c}\text { Hydroxyproline } \\
\text { release after 48h } \\
\text { (mmol/mg of protein) }\end{array}$ \\
\hline P-1 & Adult & + & 66.6 \\
2B & Animal & + & 62.0 \\
BR & Infant & W & 41.8 \\
MN & Adult & W & 34.0 \\
OE & Adult & W & 26.3 \\
HY & Adult & - & 16.0 \\
PI & Infant & - & 16.0 \\
M-I & Adult & - & 15.6 \\
DD & Animal & - & 13.5 \\
RY & Infant & - & 12.8 \\
DY & Infant & - & 10.0 \\
Bat & Environment & - & 8.7 \\
\hline
\end{tabular}

+ , Positive; -, negative; W, weak.

more frequently possessed hydrolytic enzymes and more actively degraded substrates then the less virulent strains (tables III and IV).

The ability to depolymerise chondroitin-4-sulphate and hyaluronic acid relies on the action of more than one enzyme. The release of unsaturated disaccharide-containing intermediates is catalysed by either a chrondroitin-4-sulphate or hyaluronic lyase. The subsequent breakdown of these intermediates is accomplished by the activity of $\beta$ glucuronidase to release $\mathrm{N}$-acetylhexosamine sugars and, in the case of chondroitin-4-sulphate, organic sulphate via the action of chondrosulphatase. Some of the isolates from infants performed only the first step in the degradation of these substrates leading to the accumulation of unsaturated disaccharide-containing intermediates. They clearly lack $\beta$-glucuronidase and fail to release $\mathbf{N}$ acetylhexosamine sugars. Strains possessing this enzyme, when grown in co-culture with any of these infant isolates, were, as expected, capable of degrading the disaccharide. Interestingly, co-culture of these infants, strains with any of the strains incapable of degrading either hyaluronic acid or chondroitin-4-sulphate failed to degrade the released unsaturated intermediates. This implies that these negative strains not only lack the lyase, rendering them incapable of producing the disaccharide, but also lack $\beta$-glucuronidase.

If these enzymes are important virulence factors then it is necessary to understand how they might contribute to colonisation or disease production, or both. The majority of these enzymes act against essential components of connective tissues, namely collagen and proteoglycans. If these enzymes were 
produced in vivo, it is feasible that they could contribute to tissue damage in the gut.

Hyaluronidase activity has been implicated in adhesion and dissemination of Treponema pallidum (Fitzgerald and Repesh, 1987). The enzyme degrades the surface coating of hyaluronic acid allowing direct contact between the organism and cell constituents facilitating binding via specific ligands (Peterson et al., 1983). A similar system may operate with $C$. difficile. Furthermore, the action of such enzymes would lead to the release of potentially important nutrients. For example, exogenous proline is required by the organism (Ionesco, 1978) and some strains of $C$. difficile were shown here to produce hydroxyproline from collagen. Most interestingly, Wilson and Perini (1988) found in a chemostat model of the mouse caecum that $C$. difficile utilised $\mathrm{N}$-acetylglucosamine but lacked the enzymes necessary to cleave this monosaccharide from oligosaccharide side chains. They concluded that $C$. difficile colonised poorly in the

\section{REFERENCES}

Aminoff D, Morgan W T J, Watkins W M 1925 Studies in immunochemistry: the action of dilute alkali on the $\mathrm{N}$ acetylhexosamines and the specific blood group mucoids. Biochemistry Journal 51 : 379-389.

Bornstein M B 1958 Reconstituted rat tail collagen used as substrate for tissue cultures on coverslips in Maximow slides and roller tubes. Laboratory Investigation 7: 134-137.

Borriello S P, Larson H E 1981 Antibiotics and pseudomembranous colitis. Journal of Antimicrobial Chemotherapy 7: Suppl A : 53-65.

Borriello S P et al. 1987 Clostridium difficile-a spectrum of virulence and analysis of putative virulence determinants in the hamster model of antibotic-associated colitis. Journal of Medical Microbiology 24: 53-64.

Borriello S P, Davies H A, Barclay F E 1988 a Detection of fimbriae amongst strains of Clostridium difficile. FEMS Microbiology Letters 49: 65-67.

Borriello S P, Welch A R, Barclay F E, Davies H A $1988 b$ Mucosal association by Clostridium difficile in the hamster gastrointestinal tract. Journal of Medical Microbiology 25: 191-196.

Borriello S P, Davies H A, Kamiya S, Reed P J, Seddon S Virulence factors of Clostridium difficile. Reviews of Infectious Diseases (in press).

Czuprynski C J, Johnson W J, Balish E, Wilkins T 1983 Pseudomembranous colitis in Clostridium difficile monoassociated rats. Infection and Immunity 39: 1368-1376.

Fitzgerald T S, Repesh L A 1987 The hyaluronidase associated with Treponema pallidum facilitates treponemal dissemination. Infection and Immunity 55: 1023-1028.

George $\mathrm{R} \mathrm{H}$ et al. 1978 Identification of Clostridium difficile as a cause of pseudomembranous colitis. British Medical Journal $1: 695$. model because it failed to compete for this sugar with the remaining microflora. Nevertheless, $C$. difficile can successfully colonise gnotobiotic rats (Czuprynski et al., 1983) suggesting that they may be capable of releasing this sugar from other sources since it is not found as a free monosaccharide in nature. The findings presented here offer an explanation for this observation in that some of the C. difficile isolates tested, primarly the more virulent strains, released this sugar from hyaluronic acid. However, it is also possible that, in the absence of a competing flora, other, preferential, nutrients are available.

Clearly more work needs to be performed to elucidate the role of these hydrolytic enzymes in the pathogenesis of $C$. difficile infection. However, a combination of the variable production of such enzymes and known differences in the ability to associate with the gut mucosa (Borriello et al., $1988 b$ ) might help explain why not all toxigenic strains are equally virulent.

Hafiz S 1974 Clostridium difficile and its toxins. PhD Thesis. University of Leeds.

Ionesco H 1978 Initiation de la germination des spores de Clostridium difficile par le lysozyme. Comptes Rendus Academie des Sciences [D] (Paris) 287: 659-661.

Larson H E, Price A B, Honour P, Borriello S P 1978 Clostridium difficile and the aetiology of pseudomembranous colitis. Lancet 1 : 1063-1066.

Libby J M, Wilkins T D 1982 Production of antitoxins to two toxins of Clostridium difficile and immunological comparison of the toxins by cross-neutralization studies. Infection and Immunity 35: 374-376.

Peterson K M, Baseman J B, Alderete J F 1983 Treponema pallidum receptor binding proteins interact with fibronectin. Journal of Experimental Medicine 157: 1958-1970.

Riley T V 1987 A simple method for detecting heparinaseproducing bacteria. Letters in Applied Microbiology 4: 2123.

Steffen E K, Hentges D J 1981 Hydrolytic enzymes of anaerobic bacteria isolated from human infections. Journal of Clinical Microbiology 14: 153-156.

Taylor N S, Thorne G M, Bartlett J G 1981 Comparison of two toxins produced by Clostridium difficile. Infection and Immunity 34: 1036-1043.

Tipler L S, Embery G 1985 Glucosaminoglycan-depolymerizing enzymes produced by anaerobic bacteria isolated from the human mouth. Archives of Oral Biology 30: 391-396.

Wilson K H, Perini F 1988 Role of competition for nutrients in suppression of Clostridium difficile by the colonic microflora. Infection and Immunity 56: 2610-2614.

Yamagata T, Saito H, Habuchi O, Suzuki S 1968 Purification and properties of bacterial chondroitinases and chondrosulfatases. Journal of Biological Chemistry 243: 1523-1535. 